\title{
Über das Kupfersuperoxyd.
}

\author{
Von \\ L. MOSER.
}

Im folgenden wurde die Art der Einwirkung verschiedener Oxydationsmittel auf Kupfersalze untersucht und die resultierenden Endprodukte analysiert.

Es wurden dabei folgende Oxydationsmittel berücksichtigt:

I. Chlor in alkalischer Lösung.

II. Brom in alkalischer Lösung.

III. Ozon.

IV. Wasserstoffsuperoxyd.

V. Natriumsuperoxyd.

VI. Persulfate.

VII. Andere Superoxyde.

Über die Einwirkung von Ozon und von Persulfaten scheinen keine Beobachtungen vorzuliegen, während über den Einflufs der übrigen angeführten Oxydantien auf Cuprisalze sich in der Literatur verschiedene, teils widersprechende Angaben finden.

\section{Chlor in alkalischer Lösung.}

Wenn man Chlorgas in eine Suspension von Kupferhydroxyd in Kalilauge einleitet, so soll sich nach $\mathrm{K}_{\mathrm{RÜG}} \mathrm{ER}^{1}{ }^{1}$ eine rote Lösung von kupfersaurem Kali bilden, das sich leicht unter Sauerstoffentwickelung und Hinterlassung eines schwarzen Rückstandes, der aus Kupferoxyd besteht, zersetzt. Mawnow ${ }^{2}$ untersuchte ebenfalls die Einwirkung von Chlorgas auf Cuprisalze in kalialkalischer. Lösung. Er führte eine Anzahl von Versuchen aus, wobei er die

\footnotetext{
1 Angg. Ann. 62 (1844), 447.

2 Z. anorg. Chem. $32(1900), 233$.
} 
Bedingungerı dahin ünderte, dafs er einerseits mit Kalilauge von verschiedener Dichte $(1.09-1.4)$ und in verschieden grofsem Überschufs, andererseits unter Eiskühlung, bei mittlerer Temperatur und bei $100^{\circ}$ arbeitete. In keinem Falle konnte er das Auftreten einer roten Färbung beobachten. Die getrockneten und analysierten Produkte ergaben, dafs der schwarze Körper bauptsächlich Kupferoxyd, Wasser und Spuren von aktivem Sauerstoff enthielt.

Es wurde eine grölsere Anzahl von Versuchen durchgeführt, wobei Chlor als Oxydans und Natron- oder Kalilauge von verschiedener Dichte und in verschieden grolsem Überschusse vorhanden war. Während des Einleitens von Chlorgas begann nach kurzer Zeit, auch wenn unter Eiskühlung gearbeitet wurde, eine lebhafte Sauerstoffentwickelung aufzutreten und es entstand ein dunkel gefärbter Körper. Nachdem die Färbung des Produktes sich nicht mehr änderte, wurde das Einleiten unterbrochen und mit kaltem Wasser, unter Zuhilfenahme eines Rührwerkes so lange gewaschen, bis im Filtrate kein Chlor mehr nachweisbar war. Der so erhaltene Körper wurde über Schwefelsäure und Phosphorpentoxyd getrocknet. Er war von dunkelbrauner Farbe und sah unter dem Mikroskop einheitlich aus. Mit verdünnten Säuren gibt er kein Wasserstoffsuperoxyd; Kaliumpermanganatlösung wird nicht entfärbt, mit Salzsilure entwickelt er Spuren von Chlor.

Zum Zwecke der Analyse wurde das Kupfer auf elektrolytischem Wege, das Wasser in der üblichen Weise durch Erhitzen der Substanz in einem trockenen Luftstrome und durch Bestimmung der Gewichtszunahme eines mit Chlorcalcium gefüllten U-Rohres, der aktive Sauerstoff endlich nach Bunsen auf chlorometrischem Wege bestimmt.

Da es wohl möglich ist, dals vorerst ein höheres Oxyd des Kupfers von geringer Beständigkeit entsteht, so wurde, um das langwierige Trocknen zu vermeiden, in dem feuchten Produkte das Verhältnis von Kupferoxyd zum aktiven Sauerstoff bestimmt.

\begin{tabular}{c|c|c|c|c|c}
\hline $\mathrm{Nr}$. & $\begin{array}{c}\mathrm{cem} \\
\mathrm{Na}_{2} \mathrm{~s}_{2} \mathrm{O}_{3}\end{array}$ & $\begin{array}{c}\text { entspricht } \\
g\end{array}$ & $\mathrm{~g} \mathrm{Cu}$ & $\mathrm{CuO}: \mathrm{O}$ & $\begin{array}{c}\text { Temperatur } \\
\text { in }\end{array}$ \\
\hline 1 & 2.1 & 0.0016 & 0.6434 & $100: 1$ & 15 \\
2 & 3.15 & 0.0024 & 0.8495 & $88: 1$ & 2
\end{tabular}

Bei den folgenden Versuchen wurden die gewaschenen Niederschläge getrocknet und dann analysiert. Die Ergebnisse waren die folgenden: 


\begin{tabular}{|c|c|c|c|c|c|c|c|}
\hline \multirow{2}{*}{$\mathrm{Nr}$. } & \multirow{2}{*}{$\left|\begin{array}{cc}\mathrm{cem} \\
\mathrm{b} / 1 \mathrm{CuSO}_{4}\end{array}\right|$} & \multirow{2}{*}{$\begin{array}{l}\mathrm{cem} \\
\text { NaHO }\end{array}$} & \multirow{2}{*}{$\begin{array}{c}\text { Temp. } \\
\text { in }\end{array}$} & \multicolumn{4}{|c|}{ In 100 Teilen gefunden } \\
\hline & & & & $\mathrm{C} 11 \mathrm{O}$ & $\mathrm{O}$ & $\mathrm{H}_{2} \mathrm{O}$ & $\mathrm{Na}_{2} \mathrm{O}$ \\
\hline 1 & 50 & $\begin{array}{c}300 \\
\mathrm{D}=1.1 \\
300\end{array}$ & 15 & 96.2 & 0.02 & 1.95 & 1.9 \\
\hline 2 & ธ0 & $\begin{array}{c}D=1.14 \\
500\end{array}$ & 2 & 96.27 & 0.10 & 3.10 & 0.59 \\
\hline 3 & 50 & $\begin{array}{c}\mathrm{D}=1.4 \\
500\end{array}$ & 80 & 96.15 & $0.0 \underline{6}$ & 2.54 & 2.02 \\
\hline 4 & 50 & $\begin{array}{c}D=1.5 \\
500\end{array}$ & 15 & 95.44 & 0.08 & 2.10 & 8.10 \\
\hline 5 & 50 & $\begin{array}{c}D=1.5 \\
500\end{array}$ & 2 & - & 0.06 & - & - \\
\hline 6 & 50 & $\mathrm{D}=1.5$ & 80 & $\longrightarrow$ & 0.09 & - & - \\
\hline
\end{tabular}

Trotz langen Auswaschens gelang es nicht, alkalifreie Niederschläge zu erhalten. Die Bestimmung des Alkali wurde beim Versuch 1 und 2 direkt ausgeführt, in den anderen Fällen wurde es aus der Differenz bestimmt.

Die von KRÜGER beschriebene unbeständige rote Lösung wurde nie erhalten, und ist das Produkt der Einwirkung des Chlors auch in natronalkalischer Lösung nur Kupferoxyd. Die Entstehung eines unbeständigen Primäroxydes mit Superoxydcharakter ist nicht ausgeschlossen, jedenfalls bewirkt die Hypochloritlösung den katalytischen Zerfall desselben, so dafs als Endprodukt Kupferoxyd resultiert. Die geringen Mengen von aktivem Sauerstoff' deuten auf Spuren von unzersetztem Primäroxyd hin.

\section{Brom in alkalischer Lösung.}

Vitali $^{1}$ will die Bildung von Kupfersuperoxyd gelegentlich der Einwirkung von Natriumhypobromit auf Kupferoxydhydrat beobachtet haben und schlägt diese Reaktion zum qualitativen Nachweis geringer Kupfermengen vor. Auch in diesem Falle ist die Bildung dieses Körpers recht zweifelhaft, da, wie PICcINI ${ }^{2}$ gezeigt hat, ein echtes Superoxyd niemals durch Chlor oder Brom in alkalischer Lösung entstehen kann.

Die Versuche wurden mit Bromnatronlauge von verschiedener Dichte und in verschieden grolsem Überschusse durchgeführt. Bei der Einwirkung des Hypobromits bildete sich ein schwarz gefürbter Körper und es fand Entwickelung von Sauerstoff statt, die je nach

1 Boll. Chim. Farm. 38 (1894), 668.

2 Z. anorg. Chem. 12 (1896), 169. 
der Konzentration der Lauge stärker oder schwächer war. Durch oftmaliges Dekantieren mit kaltem Wasser wurde nun das schwarze Produkt so lange gewaschen, bis sich in der ablaufenden Flüssigkeit kein Brom und kein Alkali mehr vorfand. Beim Lösen des Körpers in verdünnten Säuren konnte aber dennoch ein geringer Bromgehalt festgestellt werden. Es scheint hier im geringen Malse eine Komplexbildung stattzufinden, welche das Entstehen eines schwerlöslichen Salzes bewirkt. Durch Behandlung mit verdünnter Schwefelsäure entsteht keine Spur Wasserstoffsuperoxyd, aus Salzsäure wurde kein Chlor in Freiheit gesetzt.

Bei den folgenden Versuchen wurde das feuchte Produkt nach dem Verfahren von Bunses auf seinen Gehalt an aktivem Sauerstoff untersucht und das Kupfer durch Elektrolyse abgeschieden.

Die Verhältniszahlen $\mathrm{CuO}: \mathrm{O}$ waren die folgenden:

\begin{tabular}{c|c|c|c|c}
\hline \hline Nr. & $\begin{array}{c}\text { ccm } \\
\text { n/1 CuSO }\end{array}$ & $\begin{array}{c}\text { ccm } \\
\mathrm{NaOBr}\end{array}$ & $\begin{array}{c}\text { Temperatur } \\
\text { in }\end{array}$ & $\mathrm{CuO}: 0$ \\
\hline 1 & 50 & $\begin{array}{c}300 \\
\mathrm{D}=1.1\end{array}$ & 15 & $73: 1$ \\
2 & 50 & $\begin{array}{c}500 \\
5=1.4\end{array}$ & 15 & $79: 1$ \\
3 & 50 & $\mathrm{D}=1.1$ & 2 & $75: 1$
\end{tabular}

Bei den folgenden Versuchen wurde Kupferhydroxyd verwendet, das in Form einer feinen Suspension in Wasser vorhanden war.

\begin{tabular}{|c|c|c|c|c|}
\hline Nr. & $\begin{array}{c}\mathrm{ccm} \\
\mathrm{Cu}(\mathrm{OH})_{2} \\
\end{array}$ & $\begin{array}{c}\mathrm{ccm} \\
\mathrm{NaOBr}\end{array}$ & $\begin{array}{l}\text { Temperatur } \\
\text { in } 0\end{array}$ & $\mathrm{CuO}: \mathrm{O}$ \\
\hline 1 & 50 & $\begin{array}{c}300 \\
\mathrm{D}=1.1 \\
500\end{array}$ & 15 & $64: 1$ \\
\hline 2 & 50 & $\begin{array}{c}D=1.4 \\
300\end{array}$ & 15 & $75: 1$ \\
\hline 3 & 50 & $\begin{array}{c}\mathrm{D}=1.1 \\
300\end{array}$ & 1 & $68: 1$ \\
\hline 4 & 50 & $\mathrm{D}=1.1$ & 80 & $95: 1$ \\
\hline
\end{tabular}

Es entsteht demnach kein höheres Oxyd des Kupfers und ist die Angabe von Vitali unrichtig. Sehr wahrscheinlich kommt es auch hier zur primären Bildung eines höheren Oxyds, dessen Zerfall mit hoher Reaktionsgeschwindigkeit zur schliefslichen Bildung von Kupferoxyd führt. Die Bruttoreaktion wäre somit nach folgendem Schema darzustellen:

$$
2 \mathrm{Cu}(\mathrm{OH})_{2}+2 \mathrm{NaOBr}=2 \mathrm{CuO}+2 \mathrm{NaBr}+2 \mathrm{H}_{2} \mathrm{O}+\mathrm{O}_{2} .
$$




\section{Ozon.}

Ozon wirkt auf Cuprilösungen nicht oxydierend ein. Es wurden eine Reihe von Versuchen angestellt, indem zuerst in der Kälte, dann in der Wärme gearbeitet wurde, wobei die Lösungen neutral und alkalisch waren. Dieses negative Verhalten des Ozons ist wohl erklärlich, da sein Oxydationsdruck, infolge der geringen Anzahl freier Sauerstoffatome ein kleinerer ist als jener von den meisten Oxydationsmitteln, bei welchen der Sauerstoff im statu nascendi einwirkt.

\section{Wasserstoffsuperoxyd.}

L. Thenari ${ }^{1}$ hat 1818 zum ersten Male die Einwirkung von Wasserstoffsuperoxyd auf Kupfersalze studiert. Er wendete es in sehr verdünntem Zustande in geringem Überschusse in alkalischer Lösung an und erhielt einen olivengrünen Körper, der sich unter Sauerstoffentwickelung rasch zersetzte. Er gibt an, dals der Körper etwa zweimal so viel Sauerstoff als Kupfer enthalte. C. Weltzien ${ }^{2}$ fügte Wasserstoffsuperoxyd zu einer Lösung ron Kupferammonsulfat und bekam ebenfalls einen olivengrünen Niederschlag, den er an der Luft trocknete. Nach einer Analyse, bei der Kupferoxyd und Wasser direkt, der Sauerstoff aus der Differenz bestimmt wurde, schliefst er auf die Zusammensetzung von $\mathrm{CuO}_{2} \cdot \mathrm{H}_{2} \mathrm{O}$. Er fand ferner, dals durch Einwirkung von verdünnter Salzsäure auf das Superoxyd kein Chlor, sondern Wasserstoffsuperoxyd und Sauerstoff entstehe. W. SchMrD ${ }^{3}$ behauptete, dafs die Bildung dieses Körpers nur dann erfolge, wenn das zur Anwendung gelangende Cuprisalz eisenhaltig ist. G. KRÜss ${ }^{4}$ schüttelte fein verteiltes Kupferhydroxyd mit überschüssigem Wasserstoffsuperoxyd bei $0^{0}$ und liels es damit durch 5 Tage in Berührung. Er erhielt ebenfalls einen olivengrün gefärbten Niederschlag, der mit Alkohol und Äther gewaschen wurde und im trockenen Zustande der annähernden Zusammensetzung $\mathrm{CuO}_{2} \cdot \mathrm{H}_{2} \mathrm{O}$ entsprach. $\mathrm{Zu}$ bemerken wäre, dafs der Sauerstoff wieder aus der Differenz bestimmt, und auf einen Alkaligehalt gar keine Rücksicht genommen wurde. KRÜss gibt aufserdem noch an, dafs noch eine unbeständige $Z$ wischenstufe zu existieren scheine, deren Färbung eine grasgrüne ist. Die erste direkte Bestimmung des

1 Ann. chim. phys. 9 (1818), 51.

2 Ann. 140 (1866), 207.

${ }^{3}$ Journ. prakt. Chem. 98, (1866) 136.

4 Ber. deutsch. chem. Ges. 17 (1884), 2593. 
Superoxydsauerstoffes rührt von Tu. OsBonNe her. ${ }^{1}$ Er behandelte das Kupferoxydhydrat mit Wasserstoffsuperoxyd von verschiedener Konzentration und erhielt je nach der Dauer der Einwirkung und der Menge desselben verschieden gefärbte Produkte (von grasgrün bis braun). Er löste die noch feuchten Körper in verdünnter Schwefelsäure und bestimmte das gebildete Wasserstoffsuperoxyd durch eine Permanganatlösung. Dabei nahm er aber keine Rücksicht auf den durch Säuren gleichzeitig freigemachten Sauerstoff und er gelangte daher im günstigsten Falle zu einem Verhältnis von $\mathrm{CuO}: \mathrm{O}=1.2: 1$.

$\mathrm{Zu}$ den folgenden Versuchen wurde als Ausgangsmaterial ein Kupfersulfat oder Kupferchlorid verwendet, das vollkommen eisenfrei befunden wurde. Das Wasserstoffsuperoxyd wurde in Form des Merk schen Perhydrol verwendet und zeigte ebenfalls den erwünschten Grad von Reinheit. Die zur Herstellung des Kupferhydroxyds benutzte Kali- oder Natronlauge war ebenfalls frei von Eisen, die Fällung des Kupfersalzes geschah in der Kälte, wobei die Lauge im ganz geringen Überschufs vorhanden war. Das Auswaschen wurde durch oftmaliges Dekantieren mit kaltem Wasser durchgeführt und diese Operation so oft wiederholt, bis kein $\mathrm{SO}_{4}{ }^{\prime \prime}$ oder $\mathrm{Cl}^{\prime}$ im Filtrate nachweisbar war. ${ }^{2}$

Fügt man zu einer n/2-Kupfersulfatlösung Wasserstoffsuperoxyd von $3 \%$ hinzu, so färbt sich die Flüssigkeit gelbgrün und sieht im auffallenden Lichte trübe aus, ohne dals sich, auch bei längerem Stehen, ein unlösliches Produkt abscheiden würde. Wendet man $30 \%$ iges Wasserstoffsuperoxyd an, so fällt sofort ein gelbgrüner Niederschlag heraus und es entweicht Sauerstoff, jedoch zeigt auch in diesem Falle die über dem Niederschlag befindliche Flüssigkeit die Farbe des Cupriions. Der Vorgang lärst sich durch folgendes Schema darstellen:

$$
\mathrm{Cu}^{\prime \prime}+\mathrm{H}_{2} \mathrm{O}_{2} \rightarrow \mathrm{CuO}_{2}+2 \mathrm{H}^{\prime} \text {. }
$$

Die im Sinne von links nach rechts verlaufende Reaktion wird bald durch den entgegengesetzten Vorgang begrenzt, indem die Konzentration der entstehenden H-Ionen die weitere Bildung von Kupfersuperoxyd hindert. Fügt man nun, um die schädliche Wirkung derselben aufzuheben, ein Alkali hinzu, so findet stürmische Sauerstoffentwickelung statt, indem das Hydroperoxyd in der alkalischen

1 Chem. Nows 50 (1887), 263.

2 'Trotzdem läist sich das Alkali nicht vollkommen auswaschen und rührt daher der Alkaligehalt des Kupfersuperoxydes davou her. 
Lösung durch das Kupfersuperoxyd katalytisch zersetzt wird, wie dies ja auch bei vielen anderen höheren Oxyden $\left(\mathrm{MnO}_{2}, \mathrm{PbO}_{2}\right)$ der Fall ist.

Es ergibt sich daher aus dieser Überlegung, dals die günstigsten Bedingungen, um das Gleichgewicht möglichst auf die rechte Seite der Gleichung zu verlegen, eine neutrale Lösung sein wird und um das Entstehen von freier Säure zu verhindern, Kupferhydroxyd als Ausgangsprodukt zu wählen ist. Aufserdem ist es günstig, die Temperatur möglichst nahe dem 0-Punkte zu wählen, um den Zerfall des Kupfersuperoxyds zu verzögern.

Die Darstellung des Kupfersuperoxyds geschah auf folgende Weise: In das im Wasser fein verteilte, abgekühlte Kupferoxydhydrat wurde unter Umrühren Hydroperoxyd einfliefsen gelassen, bis letzteres im geringen Überschusse vorhanden war. Während dieser Operation änderte die Suspension ihre Färbung von grünblau über braungrün bis braun, und es entstand, je nach der Konzentration des angewendeten Wasserstoffsuperoxyds $(10 \%-30 \%)$ eine märsige oder stärkere Sauerstoffentwickelung. Wenn sich die Farbe des Niederschlages nicht mehr änderte, was nach 5-10' meist der Fall war, wurde rasch abgesaugt und das Superoxyd durch öftere Dekantation mit eiskaltem Wasser gewaschen, bis Titansulfat im Filtrate keine Gelbfärbung mehr verursachte, der Niederschlag also frei von Wasserstoffsuperoxyd war. War die Hauptmenge des Hydroperoxyds vom Niederschlage einmal entfernt, so begann die Sauerstoffentwickelung aufzuhören und es konnte das Dekantieren rasch ausgeführt werden, da sich das Kupfersuperoxyd leicht und vollständig absetzte.

Das entstandene Produkt hat eine braune Farbe und sieht, unter dem Mikroskop betrachtet, krystallinisch aus. Es löst sich sehr leicht in verdünnten Säuren unter Entwickelung von Sauerstoff und Bildung von Wasserstoffsuperoxyd.

Es verlaufen demnach folgende 2 Reaktionen nebeneinander.

1. $\mathrm{CuO}_{2}+2 \mathrm{H}^{\cdot}=\mathrm{Cu}^{*}+\mathrm{H}_{2} \mathrm{O}_{2}$

2. $2 \mathrm{CuO}_{2}+4 \mathrm{H}^{\prime}=2 \mathrm{Cu}^{\prime \prime}+2 \mathrm{H}_{2} \mathrm{O}+\mathrm{O}_{2}$.

Osborne ${ }^{1}$ hat auf die Entwickelung von Sauerstoff gar keine Rücksicht genommen, daher erhält er zu niedrige Werte für den Superoxydsauerstoff. Zum Zwecke der Analyse wurden vorerst Versuche angestellt um auf gasvolumetrischem Wege zum Ziele zu ge1. . c. 
langen, indem Platinschwamm als Katalysator verwendet wurde um das entstehende Wasserstoffsuperoxyd zu zerstören und die gesamte entwickelte Sauerstoffmenge zu messen. Nun entwickelt sich zwar anfangs sehr viel Sauerstoff durch den Einfluls des fein verteilten Platins, später nimmt aber die Reaktionsgeschwindigkeit sehr ab und es dauert geraume Zeit ( $24^{\mathrm{h}}$ und länger) bis die vollkommene Zersetzung des Hydroperoxyds in der sauren Lösung ihr Ende erreicht hat. Infolge dieser langen Reaktionsdauer wird eine ganz geringe Undichtheit des Apparates oder die Diffusion des Gases durch Kautschukschläuche zu einer grolsen Fehlerquelle und gibt Veranlassung zu unrichtigen Resultaten. Auch wurde die Anwendung von Platinsol in Frage gezogen, doch ist hier die Reaktionsgeschwindigkeit noch kleiner als im anderen Falle.

Richtige Ergebnisse konnte demnach nur ein kombiniertes Verfahren bieten, das der Eigenart der beiden Vorgänge Rechnung trägt. Die Zersetzung des Superoxyds wurde im Azotometer mit verdünnter Säure vorgenommen und das Volumen des entwickelten Sauerstoffes gemessen, die Flüssigkeit wurde dann in ein Becherglas gegossen und das entstandene Hydroperoxyd mit Permanganat. lösung titriert. Das Kupfer wurde durch Elektrolyse in derselben Probe bestimmt, wobei sich das Mn als Superoxyd an der Anode abschied.

Da das Kupfersuperoxyd ein unbeständiger Körper ist, der während des Trocknens ständig Sauerstoff verliert, so wurde vorerst das Hauptgewicht auf die Bestimmung des Verhältnisses Kupferoxyd zu Superoxydsauerstoff im feuchten Produkte gelegt.

Die durch eine gröfsere Versuchsreihe gefundenen Verhältniszahlen waren die nachstehenden:

\section{(S. Tabelle, S. 129.)}

Das verwendete Wasserstoffsuperoxyd war in der Mehrzahl der Fälle $30 \%$, einigemal wurde es auch $10 \%$ verwendet, es hatte aber die Konzentrationsänderung keinen Einflufs auf das Resultat; auch war es gleichgültig, ob ein grofser oder geringer Überschuls davon vorhanden war. Die Versuche 8-11 zeigen, dals längere Berührung des Hydroperoxyds mit dem Niederschlage nicht von Nutzen ist. Diese Beobachtung steht im Widerspruche mit den Angaben von Krüss, ${ }^{1}$ der eine 5 tügige Behandlung des Kupferhydroxyds mit Wasserstoffsuperoxyd vorschlägt. 


\begin{tabular}{|c|c|c|c|c|c|c|}
\hline Nr. & $\begin{array}{c}O \text { in } g \\
\text { gasf. best. }\end{array}$ & $\underset{\mathrm{KMnO}_{4}}{\text { cem }}$ & $\begin{array}{c}\text { gefunden } \\
\text { Gesamt-0 } \\
\text { in } \mathrm{g}\end{array}$ & $\begin{array}{l}\text { gefunden } \\
\mathrm{Cu} \text { in } \mathrm{g}\end{array}$ & $\begin{array}{c}\mathrm{CuO}: \mathrm{O} \\
\text { akt. }\end{array}$ & Anmerkung \\
\hline 1 & 0.0061 & 50.45 & 0.0738 & 0.3190 & $1.08: 1$ & \multirow{14}{*}{$\begin{array}{c}\text { Eiskühlung, } \\
\text { das } \mathrm{H}_{2} \mathrm{O}_{2} \text { blieb } 5-13^{\prime} \\
\text { lang in Berührung mit } \\
\text { dem Kupfersuperoxyd } \\
\text { Eiskühlung, } \\
\text { das } \mathrm{H}_{2} \mathrm{O}_{2} \text { blieb bei } \\
8-101^{\mathrm{h}} \text {, bei } 11 \\
\text { dureh } 24^{\text {h }} \text { dabei } \\
\text { Temperatur } 8-15^{0} \text {, } \\
\text { das } \mathrm{H}_{2} \mathrm{O}_{2} \text { wurde } \\
\text { nach } \check{5}^{\prime} \text { abgesaugt }\end{array}$} \\
\hline 2 & 0.0027 & 43.3 & 0.0389 & 0.1752 & $1.13: 1$ & \\
\hline 3 & 0.0168 & 38.3 & 0.0489 & 0.2150 & $1.1: 1$ & \\
\hline 4 & 0.0068 & 15.5 & 0.0276 & 0.1150 & $1.04: 1$ & \\
\hline 5 & 0.0051 & 40.5 & 0.0390 & 0.1594 & $1.03: 1$ & \\
\hline 6 & 0.0098 & 62.3 & 0.0062 & 0.2945 & $1.19: 1$ & \\
\hline 7 & 0.0030 & 25.40 & 0.0368 & 0.1595 & $1.08: 1$ & \\
\hline 8 & 0.0040 & 38.15 & 0.0360 & 0.1774 & $1.22: 1$ & \\
\hline 9 & 00015 & 78.7 & 0.0673 & 0.3232 & $1.2: 1$ & \\
\hline 10 & 0.0071 & 109.5 & 0.1542 & 0.7416 & $1.16: 1$ & \\
\hline 11 & 0.0016 & 44.6 & 0.0576 & 0.2938 & $1.28: 1$ & \\
\hline 12 & 0.0039 & 88.0 & 0.1222 & 0.5730 & $1.17: 1$ & \\
\hline 13 & 0.0015 & 76.9 & 0.0658 & 0.3020 & $1.15: 1$ & \\
\hline 14 & 0.0027 & 38.5 & 0.0350 & 0.1658 & $1.20: 1$ & \\
\hline
\end{tabular}

Wurde keine Rücksicht auf den durch Säuren freigemachten Sauerstoff genommen, sondern das Wasserstoffsuperoxyd direkt mit Permanganat bestimmt, so erhielt ich folgende Zahlen, welche durchschnittlich geringere Sauerstoffwerte ergaben:

\begin{tabular}{l|l|l|c|c|c}
\hline \hline $\mathrm{Nr}$. & $\begin{array}{c}\mathrm{ccm} \\
\mathrm{KMnO}_{4}\end{array}$ & $\begin{array}{c}\text { entspricht } \\
\mathrm{O} \text { in g }\end{array}$ & $\begin{array}{c}\text { gefunden } \\
\mathrm{Cu} \text { in } \mathrm{g}\end{array}$ & $\mathrm{CuO}:$ O akt. & Anmerkung \\
\hline \hline 1 & 36.5 & 0.0305 & 0.1489 & $1.2: 1$ & | Dieselb. Versuchs- \\
2 & 39.65 & 0.0664 & 0.1825 & $1.3: 1$ & | cedingungen wie \\
3 & 23.5 & 0.0196 & 0.0993 & $1.26: 1$ & früher bei Nr. 1-8 \\
4 & 43.65 & 0.0365 & 0.2257 & $1.5: 1$ & Trockenes Produkt
\end{tabular}

Es erscheint durch die angestellten Versuche als sicher festgestellt, dafs das Atomverhältnis vom Kupfer: Superoxydsauerstoff wie $1: 1$ ist. Wegen der Zersetzlichkeit des feuchten Produktes konnten trotz Eiskühlung und möglichst rascher Analyse nur Grenzwerte erreicht werden, welche in einigen Fällen dem obigen Ver. hältnisse sehr nahe kamen. Tiefere Temperaturen als $0^{\circ} \mathrm{zu}$ wählen ist nicht möglich, weil das als Suspension vorhandene Kupferhydroxyd zu Klumpen erstarrt und dadurch eine innige Berührung mit dem Wasserstoffsuperoxyd verhindert.

Eine Anzahl von den in feuchtem Zustande untersuchten Produkten wurde über Schwefelsäure und Phosphorpentoxyd im Exsiccator getrocknet. Bei der Bestimmung des Atomverhältnisses zwischen Kupferoxyd und Sauerstoff zeigte es sich, dafs der Gehalt 
an Superoxydsauerstoff in der feuchten Substanz rasch zurückging, später aber langsam, jedoch stetig abnahm. Um den Trockenprezefs zu beschleunigen, wurde in mehreren Fällen nach dem Waschen mit Wasser und möglichst vollständigem Absaugen desselben mit wenig Alkohol nachgewaschen und dieser sofort durch Äther verdrängt. Die Ätherdämpfe wurden durch Absaugen im Vakuum möglichst entfernt.

Ein mit Alkohol und Äther gewaschenes und getrocknetes Produkt ergab die nachstehenden Zahlen, welche wegen besserer Übersicht alle auf eine Einwage von $0.5 \mathrm{~g}$ Substanz berechnet wurden. Die Angaben über den volumetrisch gefundenen Sauerstoff wurden auf eine Temperatur von $15^{\circ}$ und einen Barometerstand von $760 \mathrm{~mm}$ reduziert.

\begin{tabular}{|c|c|c|c|c|c|c|}
\hline \multirow[b]{2}{*}{$\mathrm{Nr}$} & \multirow{2}{*}{$\begin{array}{c}\text { Ana- } \\
\text { lysiert } \\
\text { n. Tagen }\end{array}$} & \multirow{2}{*}{$\stackrel{\mathrm{cem}}{\mathrm{O}}$} & \multirow{2}{*}{$\begin{array}{c}\mathrm{cem} \\
\mathrm{KMnO}_{4}\end{array}$} & \multicolumn{2}{|c|}{ In 100 Teilen sind } & \multirow{2}{*}{$\begin{array}{l}\text { Atomverh. } \\
\mathrm{CuO}: 0 \text { akt. }\end{array}$} \\
\hline & & & & Ges. $O$ in $g$ & $\mathrm{CuO}$ in $\mathrm{g}$ & \\
\hline 1 & sofort & 1.1 & 76.9 & - & $\ldots$ & $1.15: 1$ \\
\hline 2 & 2 & 3.8 & 40.33 & 10.57 & 72.31 & $1.37: 1$ \\
\hline 3 & 3 & 5.4 & 32.41 & 10.03 & 73.71 & $1.47: 1$ \\
\hline 4 & 4 & 5.8 & 31.80 & 10.08 & 73.66 & $1.46: 1$ \\
\hline 5 & 8 & 6.2 & 29.68 & 9.92 & 73.90 & $1.49: 1$ \\
\hline 6 & 16 & 8.4 & 27.6 & 9.64 & 74.09 & $1.55: 1$ \\
\hline 7 & 28 & 11.7 & 22.38 & 9.14 & 74.23 & $1.63: 1$ \\
\hline 8 & 36 & 8.09 & 25.28 & 8.95 & 74.27 & $1.67: 1$ \\
\hline 9 & 72 & 8.8 & 23.95 & 8.76 & 75.24 & $1.73: 1$ \\
\hline 10 & 92 & 8.4 & 23.95 & 8.66 & 75.47 & $1.75: 1$ \\
\hline
\end{tabular}

Interessant ist die Tatsache, dals die durch Säuren aus dem Kupfersuperoxyd freigemachte Sauerstoffmenge in dem Mafse langsam wächst, als der stetige Zerfall des Peroxyds fortschreitet. Bei der Untersuchung des feuchten, also möglichst unzersetzten Produktes erhielt ich stets, wie auch aus dem Versuch 1 der obigen Reihe hervorgeht, sehr wenig gasförmigen Sauerstoff $(1-3 \mathrm{ccm})$.

Das Volumen des so entwickelten Sauerstoffs kann als Kriterium für die Reinheit des Kupfersuperoxyds angesehen werden. Während der langsamen Zersetzung desselben wächst die durch Säuren entstehende Menge Sauerstoff bis zu einer gewissen Grenze - es scheint dabei das Peroxyd einen mehr unechten Charakter anzunehmen - (bis Nr. 7 in obiger Tabelle), um dann parallel mit dem Gesamtoxydationswert abzunehmen. Vielleicht entsteht dabei eine sehr unbeständige Zwischenstufe, welche die Brücke zum letzten Zerfallsprodukt, dem Kupferoxyd bildet. Dieser Übergang spricht wieder 
dafür, dafs ein tiefgehender Unterschied zwischen Ozoniden und Antozoniden nicht anzunehmen ist und dals der Konzentration, eine wichtige Rolle zukommt. Eine weitere Stütze für diesen Übergangscharakter bietet die Tatsache, dafs das mit konzentrierter Salzsäure behandelte Superoxyd so viel Chlor aus ihr in Freiheit setzt, das es etwa dem dritten Teile des Gesamtsauerstoffgehaltes entspricht.

Zwei Versuche, welche mit einem getrockneten Superoxyde angestellt wurden, hatten folgendes Ergebnis:

\begin{tabular}{|c|c|c|c|c|c|c|}
\hline \multirow{2}{*}{$\mathrm{Nr}$} & \multirow{2}{*}{$\begin{array}{l}\text { Einwage } \\
\text { in } g\end{array}$} & \multirow{2}{*}{$\begin{array}{l}\text { verbraucht } \\
\text { ecm } \\
\mathrm{Na}_{2} \mathrm{~S}_{2} \mathrm{O}_{3}\end{array}$} & \multicolumn{2}{|c|}{ entspricht } & \multirow{2}{*}{$\begin{array}{c}\text { wirklicher } \\
\text { Gehalt } \\
\text { o akt. in } \% \\
\end{array}$} & \multirow{2}{*}{ Anmerkung } \\
\hline & & & $\mathrm{O}$ in $\mathrm{g}$ & $0 \%$ in $\%$ & & \\
\hline 1 & 1.0000 & 35.6 & 0.0285 & 2.85 & 8.88 & $\mathrm{HCl}$ \\
\hline 2 & 0.2554 & 11.2 & 0.0090 & 3.52 & 11.02 & $\mathrm{HCl}(\mathrm{D}=1.19)$ \\
\hline
\end{tabular}

Dasselbe Produkt, mit sehr verdünnter Salzsäure (n/2-) behandelt, lieferte, wie immer, Sauerstoff in geringer Menge und Wasserstoffsuperoxyd.

Die Untersuchung einer Anzahl getrockneter Produkte, welche nur mit Wasser gewaschen wurden, ergab folgende Werte:

\begin{tabular}{|c|c|c|c|c|c|c|c|}
\hline \multirow{2}{*}{ Nr. } & \multirow{2}{*}{ Einwage } & \multirow{2}{*}{$\stackrel{\mathrm{ccm}}{0}$} & \multirow{2}{*}{$\begin{array}{c}\mathrm{cmn} \\
\mathrm{KMnO}_{4}\end{array}$} & \multirow{2}{*}{$\begin{array}{l}\text { Gesamt- } \\
O \text { akt. } \\
\text { in } g\end{array}$} & \multicolumn{2}{|c|}{ In 100 Teilen } & \multirow{2}{*}{$\begin{array}{c}\overline{\text { Atomverhält }} \\
\text { nis } \\
\mathrm{CuO}: \mathrm{O}\end{array}$} \\
\hline & & & & & $\mathrm{CuO}$ & 0 akt. & \\
\hline 1 & 0.5492 & 10.60 & 46.59 & 0.0682 & 72.15 & 9.66 & $1.5: 1$ \\
\hline 2 & 0.7796 & 12.2 & 46.5 & 0.0786 & 76.60 & 10.08 & $1.52: 1$ \\
\hline 3 & 0.7670 & 22.8 & 29.0 & 0.0530 & 75.55 & 8.88 & $1.72: 1$ \\
\hline
\end{tabular}

Die folgende Versuchsreihe stammt von Produkten her, welche mit Aikohol und Äther gewaschen worden waren und daỏurch rascher getrocknet werden konnten. Sie weisen durchschnittlich einen etwas höheren Sauerstoffgehalt auf. ${ }^{1}$

\begin{tabular}{c|c|c|c|c|c|c|c}
\hline \hline Nr. & Einwage & $\begin{array}{c}\mathrm{ccm} \\
\mathrm{O}\end{array}$ & $\begin{array}{c}\mathrm{ccm} \\
\mathrm{KMnO}_{4}\end{array}$ & $\begin{array}{c}\text { Gesamt- } \\
\text { O akt. } \\
\text { in } \mathrm{g}\end{array}$ & \multicolumn{2}{|c|}{$\begin{array}{c}\text { In } 100 \text { Teilen } \\
\mathrm{CuO}\end{array}$} & $\begin{array}{c}\text { O A akt. } \\
\text { nis } \\
\mathrm{CuO}: \mathrm{O}\end{array}$ \\
\hline \hline 1 & 0.5262 & 6.5 & 33.15 & 0.0531 & 73.66 & 10.09 & $1.46: 1$ \\
2 & 0.5253 & 5.6 & 33.70 & 0.0527 & 73.71 & 10.03 & $1.47: 1$ \\
3 & 0.5000 & 4.1 & 56.5 & 0.0531 & 73.35 & 10.62 & $1.38: 1$
\end{tabular}

Aus den angeführten Versuchen ist zu ersehen, dafs das Kupfersuperoxyd in feuchtem Zustande eine partielle Zersetzung erleidet.

1 Tatsächlich wurden in beiden Fällen mehr Versuche ausgeführt, der Kürze halber sind nur einige Durchschnittsversuche herangezogen worden. 
Wie aus der auf S. 130 angegebenen Tabelle ersichtlich ist, zeigt der Übergang aus dem feuchten Zustande in den trockenen den gröIsten Sprung in den Verhältniszahlen, im weiteren Verlaufe ist der Zerfall ein langsamer. Hätte $\mathrm{KRÜss}^{1}$ in dem getrockneten Produkte eine direkte O-Bestimmung vorgenommen, so wäre er unmöglich zu den fast theoretischen Sauerstoffwerten gelangt.

Wegen der erwähnten Zersetzlichkeit ist es schwierig, die Bestimmung des chemisch gebundenen Wassers vorzunehmen. Hat man nur mit Wasser gewaschen und trocknet man über Schwefelsäure oder Phosphorpentoxyd, so dauert es geraume Zeit bis die Substanz von der mechanisch anhaftenden Feuchtigkeit befreit ist. Während dieser Zeit findet nun, wie gezeigt wurde, eine teilweise Zersetzung statt. Wäscht man mit Alkohol und Äther, so wird der Trockenprozefs zwar bedeutend verkürzt, es haften jedoch die letzten Spuren von Äther sehr fest und sind auch durch längeres Evakuieren kaum vollständig $\mathrm{zu}$ entfernen.

Als nun die Substanz zum Zwecke der Wasserbestimmung in einem Rohr erwärmt wurde, fand unter Einwirkung des Ätherdampfes ein plötzlicher Zerfall des Kupfersuperoxydes statt, der bäufig die Analysensubstanz bis in das zur Aufnahme des Wassers bestimmte U-Rohr schleuderte. Einigemale war diese Zersetzung von einer Lichterscheinung begleitet.

Auch bei gewöhnlicher Temperatur können Spuren von Äther einen plötzlichen Zerfall des Superoxyds herbeiführen. Diese Beobachtung wurde gemacht, als eine gröfsere Menge trockenes Kupfersuperoxyd, das nicht im mindesten mehr nach Äther roch, mit einem Platinspatel auf einem Uhrglase zerrieben wurde. Es kam dabei zu einer ungefährlichen Explosion, der Rückstand war nur mehr Kupferoxyd.

Unter der Voraussetzung, dafs, falls die Substanz überhaupt Wasser enthält, eine chemische Bindung desselben vorliegt, mufs beim Zerfall die Sauerstoff- und Wasserabgabe parallel verlaufen, und das Atomverhältnis des gefundenen Wassers zum Sauerstoff ein einfaches sein. Da nun im feuchten Körper der Sauerstoffgehalt zum Kupferoxyd sich wie 1:1 verhält, in dem trockenen Produkte sich dieses Verhältnis zuungunsten des Sauerstoffs verschiebt, so muls sich derselbe Vorgang auch für Wasser abspielen und die Verhältniszablen zwischen letzterem und dem Sauerstoff wieder wie $1: 1$ oder ein Vielfaches davon sein.

1 l. e. 
Es wurde deshalb in der Weise verfahren, dafs die kohlensäurefreie Luft zuerst vorgewärmt und dann über das in einem Verbrennungsrohr befindliche Kupfersuperoxyd, welches zwischen zwei Asbestpfropfen lag, darübergeleitet wurde. Erst nach einiger Zeit wurde mälsig erwärmt, schliefslich geglüht und das Wasser durch Chlorcalcium aufgefangen.

\begin{tabular}{|c|c|c|c|c|}
\hline \multirow{2}{*}{$\mathrm{Nr}$} & \multicolumn{2}{|c|}{ In 100 Teilen gefunden } & \multirow{2}{*}{$\begin{array}{c}\text { Atomverhält- } \\
\text { nis } \\
\mathrm{H}_{2} \mathrm{O}: \mathrm{O}\end{array}$} & \multirow{2}{*}{ Anmerkung } \\
\hline & 0 & $\mathrm{H}_{2} \mathrm{O}$ & & \\
\hline 1 & 10.54 & 11.98 & $1.01: 1$ & mit Alk. + Äther gewaschen \\
\hline 2 & 11.22 & 11.23 & $1: 1.12$ & $"$ \\
\hline 3 & 9.60 & 12.02 & $1.11: 1$ & nur mit Wasser gewaschen \\
\hline 4 & 10.94 & 11.48 & $1: 1.07$ & mit Alk. + Äther gewaschen \\
\hline$\tilde{5}$ & 8.90 & 10.89 & $1.08: 1$ & nur mit Wasser gewaschen \\
\hline
\end{tabular}

Aus den gewonnenen Zahlen darf man den Schlufs ziehen, dafs das Kupfersuperoxyd ein Molekül Wasser im unzersetzten Zustande enthält.

Aus den gesamten Untersuchungen geht demnach hervor, dafs die Formel des Kupfersuperoxyds $\mathrm{CuO}_{2} \cdot \mathrm{H}_{2} \mathrm{O}$ ist.

Eigenschaften:

Kupfersuperoxyd ist im reinen Zustande ein brauner, krystallinischer Körper, im feuchten Zustande rasch zersetzlich, im trockenen findet ein langsamer Zerfall unter Abgabe von Wasser und Sauerstoff zu Kupferoxyd statt, in Wasser unlöslich, beim Kochen mit Wasser entwickelt sich Sauerstoff, die Flüssigkeit reagiert neutral. In verdünnten Säuren leicht löslich unter Bildung von Wasserstoffsuperoxyd und wenig Sauerstoff, in konzentrierter Salzsäure unter Chlorentwickelung und Abgabe von Sauerstoff löslich, mit Alkalien setzt es sich um unter Bildung von Hydroxyd and Sauerstoffentwickelung.

Kaliumpermanganatlösung wird entfärbt, in alkalischer und neutraler Lösung wirkt es katalytisch auf Wasserstoffsuperoxyd ein. Es verhält sich gröfstenteils wie ein echtes Superoxyd, was der Verwandschaft des Kupfers zu den Alkalien entsprechend ist. Im unreinen Zustande ist es olivengrün bis grasgrün gefärbt (enthält noch Kupferhydroxyd).

\section{v. Natriumsuperoxyd.}

Die Einwirkung von Natriumsuperoxyd auf Cuprisalze wurde bisher nur von B. C. BrodiE ${ }^{1}$ untersucht. Er erkannte, dals bei

1 Jahresber. 1862, 115. 
Zusatz dieses Körpers zu einer Kupferoxydsalzlösung Kupferhyperoxyd entsteht. Im folgenden wurde eine Reihe von Versuchen durchgeführt, wobei Natriumsuperoxyd und auch dessen Hydrat $\mathrm{Na}_{2} \mathrm{O}_{2} .8 \mathrm{H}_{2} \mathrm{O}$ in Anwendung kam. Letzteres wurde in der Weise hergestellt, dafs Natriumsuperoxyd in einer koblensäurefreien Atmosphäre während einiger Tage über Wasser stehen gelassen wurde. Dabei änderte sich die gelbe Farbe und es entstand das reine weifse Hydrat.

1. Versuch.

a) Natriumsuperoxyd.

Angewendet:

$$
\begin{gathered}
250 \mathrm{ccm} \quad \mathrm{n} / 2 \mathrm{CuSO}_{4}, \\
5 \mathrm{~g} \quad \mathrm{Na}_{2} \mathrm{O}_{2} .
\end{gathered}
$$

Das Superoxyd wurde unter Eiskühlung in die bewegte Kupferlösung in kleinen Partien eingetragen, wobei lebhaftes Aufschäumen der Flüssigkeit stattfand. Die Temperatur betrug vor dem Versuch $+1^{\circ}$ und stieg im Verlaufe desselben auf $19^{\circ}$. Es resultierte ein blaugrüner Körper, der mit Wasser so lange gewaschen wurde, bis das Filtrat keine $\mathrm{SO}_{4}$ "-Reaktion mehr zeigte. Das feuchte Produkt löste sich in verdünnter Schwefelsäure leicht auf, es fand dabei keine Gasentwickelung und keine Bildung von Wasserstoffsuperoxyd statt. Ein anderer Teil wurde im feuchten Zustande mit Salzsäure (1:1) erwärmt, im Destillate fand keine Jodausscheidung statt. Es entstand somit nur Kupferoxydhydrat.

2. Versuch.

Angewendet: $\quad 250 \mathrm{ccm} \mathrm{n} / 2 \mathrm{CuSO}_{4}$, $10 \mathrm{~g} \quad \mathrm{Na}_{2} \mathrm{O}_{2}$.

Bei diesem Versuche wurde nicht gekühlt, es fand lebhafte Sauerstoffentwickelung statt und es bildete sich sofort ein schwarzer Körper. Nach dem Waschen mit kaltem Wasser, das eine recht langwierige Operation ist, konnte kein aktiver Sauerstoff, keine Chlor- und keine Wasserstoffsuperoxydbildung wahrgenommen werden. Infolge der hohen Temperatur findet eine Anhydrisierung des Kupferhydroxyds statt und man erhält sofort das schwarze Oxyd.

1. Versuch.

b) Natriumsuperoxydhydrat.

Angewendet: $\quad 100 \mathrm{ccm} \mathrm{n} / 2 \mathrm{CuSO}_{4}$,

$5 \mathrm{~g} \quad \mathrm{Na}_{2} \mathrm{O}_{2} .8 \mathrm{H}_{2} \mathrm{O}$. 
Das Natriumsuperoxydhydrat wurde unter Eiskühlung und bei Anwendung eines Rührwerkes eingetragen, wobei mälsige Sauerstoffentwickelung stattfand und ein dunkelgrüner Körper herausfiel. Eine kleine Menge desselben wurde sofort mit eiskaltem Wasser gewaschen. Beim Lösen in verdünnter Schwefelsäure fand Sauerstoffentwickelung und Bildung von Hydroperoxyd statt, was demnach auf das primäre Entstehen von Kupfersuperoxyd deutete. Da sich infolge der Gasentwickelung das Produkt in der alkalischen Lösung nur schwierig absetzt, ferner das Auswaschen lange Zeit in Anspruch nimmt (es mulste $18 \mathrm{mal}$ dekantiert werden um das Alkali zu entfernen), so war es unmöglich, auf diese Weise eine gröfsere Menge des unzersetzten Produktes zu erhalten. Das zuerst entstandene Kupfersuperoxyd ging während dieser langwierigen Operation vollständig ins blaue Hydroxyd über, was aus der Tatsache hervorging, dafs die Hauptmenge des gewaschenen Niederschlages nicht mehr die Reaktionen des Superoxyds zeigte.

2. Versuch.

Angewendet: $\quad 100 \mathrm{ccm} \mathrm{n} / 2 \mathrm{CuSO}_{4}$,

$$
10 \mathrm{~g} \quad \mathrm{Na}_{2} \mathrm{O}_{2} .8 \mathrm{H}_{2} \mathrm{O} \text {. }
$$

Hier wurde bei Zimmertemperatur gearbeitet, die Gasentwickelung war stürmisch und es kam rasch zur Bildung des Kupferhydroxyds.

Das Verhalten des Natriumsuperoxyds zu Kupferlösungen bietet ein treffliches Beispiel für das primäre Entstehen der unbeständigsten Formen. Je nach Wahl der Versuchsbedingungen gelingt es, entweder Kupfersuperoxyd, Hydroxyd oder das Oxyd zu erhalten. Es gehen demnach folgende Reaktionen vor sich:

1. $\mathrm{CuSO}_{4}+\mathrm{Na}_{2} \mathrm{O}_{2}=\mathrm{CuO}_{2}+\mathrm{Na}_{2} \mathrm{SO}_{4}$.

2. $2 \mathrm{CuO}_{2}+4 \mathrm{NaHO}+2 \mathrm{H}_{2} \mathrm{O}=\mathrm{Cu}(\mathrm{OH})_{2}+4 \mathrm{NaHO}+\mathrm{O}_{2}$.

3. $\mathrm{Cu}(\mathrm{OH})_{2}=\mathrm{CuO}+\mathrm{H}_{2} \mathrm{O}$.

In allen Fällen entsteht zuerst das Superoxyd, aber in der alkalischen Lösung zerfällt dieser unbeständige Körper katalytisch unter Entwickelung von Sauerstoff. Die Reaktionsgeschwindigkeit nimmt mit steigender Temperatur zu, so dafs die labilen $\mathrm{Z}$ wischenstufen sehr rasch durchlaufen werden und man als Endprodukt des ganzen Vorganges Kupferoxyd erhält.

Dafs das Kupfersuperoxyd in einer alkalischen Lösung aufserordentlich rasch zersetzt wird, kann auch auf die Weise gezeigt 
werden, dafs man auf trockenes, mit Hilfe von Wasserstoffsuperoxyd hergestelltes Kupfersuperoxyd Natronlauge bei gewöhnlicher Temperatur einwirken lälst. Es findet sofort Sauerstoffentwickelung und Bildung von Kupferhydroxyd statt. Erwärmt man schwach, so erhält man sofort das Oxyd des Kupfers.

VI. Kaliumpersulfat.

Da die Überschwefelsäure und ihre Salze auf viele Körper eine oxydierende Wirkung ansüben, so wurde ihr Verhalten den Cuprisalzen gegenüber untersucht. Ditrich und $\mathrm{H}_{\text {ASSEL }}{ }^{1}$ und v. KNoRRE ${ }^{2}$ haben das Persulfat zum Zwecke analytischer Trennungen von verschiedenen Metallen empfohlen; die hierbei verwendeten Lösungen sind entweder neutral oder schwach sauer. In derartigen Lösungen bewirken Persulfate keine Fällung des Kupfers. Die Einwirkung von Kaliumpersulfat auf Cuprisalze in alkalischer Lösung ist meines Wissens noch nicht untersucht worden.

Es wurde in der Kälte eine Cuprisulfatlösung mit Kalilauge versetzt und eine konzentrierte Lösung von Kaliumpersulfat hinzugefügt. Die Temperatur betrug dabei $+14^{\circ}$. Unter Entwickelung von Sauerstoff fiel ein dunkelbraun gefärbter Niederschlag heraus. Dieser wurde mit eiskaltem Wasser durch Dekantation gewaschen, filtriert und so lange gewaschen, bis im Filtrate kein Alkali mehr nachweisbar war.

Auf diese Weise wurde bei 3 Versuchen verfahren, wobei angewendet wurden:

1. Versuch:

2. Versuch:

3. Versuch:

$200 \mathrm{ccm} \mathrm{n} / 2 \mathrm{CuSO}_{4}$

$50, \quad \mathrm{NaHO}(\mathrm{D}=1.2)$ $200 \mathrm{ccm} \mathrm{n} / 2 \mathrm{CuSO}_{4}$ $100, \quad \mathrm{~K}_{2} \mathrm{~S}_{2} \mathrm{O}_{8}$

Zimmertemperatur

$50, \quad \mathrm{KHO}(\mathrm{D}=1.2)$ $200 \mathrm{ccm} \mathrm{n} / 2 \mathrm{CuSO}_{4}$

$100, \mathrm{~K}_{2} \mathrm{~s}_{2} \mathrm{O}_{8}$

Zimmertemperatur

$30, \mathrm{NaHO}$

$150 \quad, \quad \mathrm{~K}_{2} \mathrm{~S}_{2} \mathrm{O}_{8}$

Eiskühlung.

Die Ergebnisse waren folgende:

\begin{tabular}{c|c|c|c|c|c}
\hline $\mathrm{Nr}$. & $\begin{array}{c}\text { ccm } \\
\mathrm{Na}_{2} \mathrm{~S}_{2} \mathrm{O}_{3}\end{array}$ & $\begin{array}{c}\text { entspricht } \\
\text { CuO in } \mathrm{g}\end{array}$ & $\mathrm{O}$ in $\mathrm{g}$ & $\begin{array}{c}\text { Atomverhältnis } \\
\mathrm{CuO}: \mathrm{O}\end{array}$ & Temperatur \\
\hline \hline 1 & 9.2 & 2.8125 & 0.0073 & $77: 1$ & $14^{0}$ \\
2 & 2.75 & 0.9925 & 0.0022 & $88: 1$ & $14^{0}$ \\
3 & 4.1 & 1.3866 & 0.0033 & $85: 1$ & $2^{0}$
\end{tabular}

1 Ber, deutselu. chem. Ges. 1902, 3266. 4072; 1903, 284. 1423 ferner K. Hassed, Dissertation, Heidelberg 1903.

z Zeitschr. unalyt. Chem. 43 (1904), 1. 
In verdünnter Schwefelsäure löst sich der Körper nur langsam auf, es findet dabei keine Gasentwickelung und keine Bildung von Wasserstoffsuperoxyd statt.

Auf Kupferhydroxyd wirkt Kaliumpersulfat in neutraler Lösung gar nicht ein.

Es entsteht demnach kein höher oxydiertes Produkt, der Sauerstoff des Persulfats entweicht im molekularen Zustande und wirkt auf das Cupriion nicht ein. Möglich ist es, dafs primär ein unbeständiges Peroxyd entsteht, welches sich unter dem Einflusse des überschüssigen Persulfats in der alkalischen Lösung zu Kupferoxyd unter Sauerstoffabgabe umlagert. Für diese Annahmen sprechen die geringen Mengen aktiven Sauerstoffs, welche die J-Ausscheidung bewirken.

Statt der Alkalilauge wurde auch Ammoniak verwendet, doch findet dabei gar keine Fällung statt. In Anwesenheit eines so starken Komplexions, wie es das Kupferammoniakion ist, ist die Konzentration der Cupriionen so gering, dafs trotz der Anwesenheit von Hydroxylionen das Löslichkeitsprodukt des Kupferhydroxyds nicht überschritten wird, daher kein Niederschlag herausfällt. Dem= nach bewirken Persulfate in alkalischer Lösung keine Oxydation des Cupriions.

\section{Andere Superoxyde.}

Wenn man eine Kupfersulfatlösung mit überschüssigem Mangansuperoxyd in der Kälte unter häufigen Schütteln durch längere Zeit in Berührung lälst, so bildet sich nach W. ScHMrd ${ }^{1}$ Kupfersuperoxyd, das identisch sein soll mit dem durch Einwirkung von Wasserstoffsuperoxyd auf Cuprisalze erhaltenen Körper.

Die Unmöglichkeit der Bildung dieser Verbindung ist von vornherein einzusehen, wenn man bedenkt, dals dieses Superoxyd echten Charakter aufweist, also durch ein unechtes Peroxyd nicht dargestellt, sondern zerstört werden müfste. Immerhin ist die Möglichkeit der Entstehung einer höher oxydierten Kupferverbindung vorhanden, von einer quantitativen Umsetzung, wie ScHmID eine solche nach 3 Wochen wahrgenommen haben will, kann jedoch nicht die Rede sein.

In Anwendung kam hier eine $\mathrm{n} / 10 \mathrm{CuSO}_{4}$-Lösung, als einwirkende Superoxyde wurden nach den Angaben von Schmo das des Mangans und das des Bleis versucht.

1 Journ. prakt. Chem. 98 (1866), 136. 
Die Kupfervitriollösung wurde in einem Schütteltrichter durch längere Zeit hindurch stehen gelassen, häufig durchgeschüttelt unả von Zeit zu Zeit sowohl die Flüssigkeit als der Niederschlag untersucht.

1. Versuch.

Angewendet: $\quad 100 \mathrm{ccm} \mathrm{n} / 2 \mathrm{CuSO}_{4}$,

$0.2 \mathrm{~g} \mathrm{MnO}_{2}$.

\begin{tabular}{|c|c|c|c|}
\hline \multirow{2}{*}{$\mathrm{Nr}$. } & \multirow{2}{*}{ Dauer der Einwirkung } & \multicolumn{2}{|c|}{ Es enthielt } \\
\hline & & Die Lösung & Der Niederschlag \\
\hline $\begin{array}{l}1 \\
2 \\
3\end{array}$ & $\begin{array}{ll}3 & \text { Wochen } \\
2 & \text { Monate } \\
5 & \text { Monate }\end{array}$ & $\begin{array}{l}\text { Spuren von } \mathrm{Mn} \\
\text { geringe Menge } \mathrm{Mn} \\
\text { geringe Menge } \mathrm{Mn}\end{array}$ & $\begin{array}{cl}\mathrm{Cu} \\
\text { Spur von } \mathrm{Cu} \\
\text { geringe } \text { Menge } \mathrm{Cu}\end{array}$ \\
\hline
\end{tabular}

2. Versuch.

Angewendet: $\quad 100 \mathrm{ccm} \mathrm{n} / 2 \mathrm{CuSO}_{4}$,

$1 \mathrm{~g} \quad \mathrm{MnO}_{2} \cdot \mathrm{H}_{2} \mathrm{O}$ (frisch gefällt).

\begin{tabular}{c|c|c|c}
\hline \multirow{2}{*}{ Nr. } & Dauer der Einwirkung & Die Lösung & Der Niederschlag \\
\hline 1 & 3 Wochen & geringe Menge Mn & Spuren von Cu \\
2 & 2 Monate & geringe Menge Mn & geringe Menge Cu \\
3 & 5 Monate & geringe Menge Mn & geringe Menge Cu
\end{tabular}

3. Versuch.

Angewendet: $\quad 100 \mathrm{ccm} \mathrm{n} / 10 \mathrm{CuSO}_{4}$,

$2 \mathrm{~g} \quad \mathrm{PbO}_{2}$.

\begin{tabular}{c|c|c|c}
\hline \multirow{2}{*}{ Nr. } & Dauer der Einwirkung & \multicolumn{2}{|c}{ Es enthielt } \\
\cline { 3 - 4 } & & Die Lösung & Der Niederschlag \\
\hline \hline 1 & 3 Wochen & kein $\mathrm{Pb}$ & Spuren von $\mathrm{Cu}$ \\
2 & 2 Monate & kein $\mathrm{Pb}$ & Spuren von Cu \\
3 & 5 Monate & kein $\mathrm{Pb}$ & geringe Menge von $\mathrm{Cu}$
\end{tabular}

Der Rückstand wurde in allen Fällen vorerst mit Wasser gewaschen, um ihn von der anhaftenden Kupfersulfatlösung zu befreien, dann wurde er mit sehr verdünnter Schwefelsäure behandelt, wobei kein Aufbrausen, wie dies nach ScHurd erfolgen soll, und auch keine Bildung von Wasserstoffsuperoxyd wahrgenommen wurde. Dals bei der langen Einwirkungsdauer etwas Mangan in Lösung geht, ist wohl möglich, da Kupfersulfat in wässeriger Lösung saure Reaktion zeigt. Die geringe Menge Kupfer, welche im Nieder- 
schlage nachgewiesen wurde, ist wahrscheinlich als Oxyd vorhanden, sicher nicht als Kupfersuperoxyd.

Es findet durch Einwirkung von Mangan- oder Bleisuperoxyd auf Cuprisalze keine Superoxydbildung statt.

Schliefslich sei noch einer Beobachtung Erwähnung getan, welche ebenfalls von KnÜss ${ }^{1}$ herrührt. Dieser Forscher will ein höheres Oxyd des Kupfers durch Zusammenschmelzen von Kupferoxyd mit überschüssigem Kaliumbydroxyd erhalten haben. Durch Auslaugen der Schmelze mit Wasser von $0^{0}$ erhielt er einen rötlichen Körper. Das Produkt enthielt kein Kalium und verhielt sich, wie KRÜss angibt, wie ein höheres Oxyd des Kupfers.

Wie aus den Eigenschaften des Kupfersuperoxyds hervorgeht, ist die Existenz desselben bei einer derartigen Temperatur unmöglich. Der Versuch wurde genau nach den Angaben wiederholt und dabei eine geringe Menge eines rötlichen Körpers erhalten, der aber hauptsächlich aus Kupferoxydul bestand. Das gewöhnliche Kaliumhydroxyd des Handels enthält immer Superoxyd; bei dem Schmelzprozefs wirkt dieses auf das Kupferoxyd ein und es bildet sich unter Sauerstoffentwickelung Kupferoxydul, das identisch sein dürfte mit jenem von KRÜss erhaltenen roten Produkte.

Ursprünglich bestand die Absicht, auch die anodische Oxydation von Kupfersalzen in den Kreis der Betrachtungen zu ziehen, doch wurde dieses Thema inzwischen von $\mathrm{E}$. MÜLLER und F. SPITZER ${ }^{2}$ eingehend behandelt. Als Kathode wurde Platin, als Anode Kupfer verwendet, während der Elektrolyt eine n/12-Natronlauge war. Unter entsprechender Kühlung erbielten sie ein äufserst labiles Produkt, dessen Isolierung wegen seiner grofsen Zersetzlichkeit unmöglich war. Sie kommen zu dem Resultate, dafs das Kupfer in dreiwertiger Form bei diesem Vorgang auftritt und dem Körper, im Falle er ein Oxyd ist, die Formel $\mathrm{Cu}_{2} \mathrm{O}_{3}$ zukommen würde. Jedenfalls ist dieses Oxyd nicht identisch mit dem eigentlichen Kupfersuperoxyd.

Von Interesse dürften noch die thermochemischen Daten über das Kupfersuperoxyd sein, diese sollen demnächst Gegenstand einer eingehenden Untersuchung werden.

\section{Zusammenfassung.}

Die einzige Methode, um Kupfersuperoxyd $\mathrm{CuO}_{2} \cdot \mathrm{H}_{2} \mathrm{O}$ zu erhalten, besteht in der Oxydation von fein rerteiltem Kupferoxyd-

1 Ber. deutsch. chem. Ges. 17 (1884), 2596.

${ }^{2}$ Z. f. Elektrochem. 13 (1907), 25. 
hydrat durch Wasserstoffsuperoxyd $(15-30 \%)$ in neutraler Lösung, wobei die Temperatur nabe dem 0-Punkte sein soll. Im feuchten Zustande zerfällt das Kupfersuperoxyd rasch unter Abgabe von Sauerstoff und Wasser, trocken geht dieser Zerfall nur langsam vor sich. Durch die Einwirkung von Chlor und Brom in alkalischer Lösung wird nur Kupferoxyd erhalten und sind die gegen-

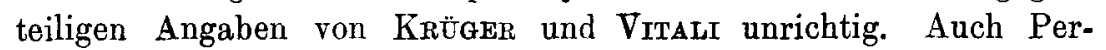
sulfate bewirken in alkalischer Lösung keine Oxydation in Cuprisalzen. Durch Natriumsuperoxyd entsteht zwar Kupfersuperoxyd, doch tritt infolge der alkalischen Flüssigkeit ein aufserordentjich rascher Zerfall desselben ein. Schlieislich ist die Beobachtung von W. ScHMrd unrichtig, der durch Behandlung von Cuprisulfat mit Mangansuperoxyd Kupfersuperoxyd erhalten haben will.

Die vorliegende Arbeit wurde im Laboratorium für analytische Chemie an der k. k. technischen Hochschule in Wien im Sommersemester 1906 begonnen und zu Ostern 1907 abgeschlossen. Dem Leiter dieses Laboratoriums, Herrn Prof. Dr. G. Vormmann, sage ich für sein stetes Entgegenkommen und das der Arbeit zugewandte Interesse meinen innigsten Dank. Ebenso fühle ich mich verpflichtet, Herrn Dr. A. Skrabal für mannigfache Ratschläge bestens zu danken.

Wien, Laboratorium für analyt. Chemie der techn. Hochschule, Ostern 1907.

Bei der Redaktion eingegangen am 6. April 1907. 